Western University

Scholarship@Western

Education Publications

Education Faculty

2016

Repetitive Behavior in Children with Down Syndrome: Functional Analysis and Intervention

Nicole Neil

WesternUniversity,nneil@uwo.ca

Emily A. Jones

Queens College

Follow this and additional works at: https://ir.lib.uwo.ca/edupub

Part of the Education Commons, and the Psychology Commons

Citation of this paper:

Neil, N., \& Jones, E. A. (2016). Repetitive behavior in children with Down Syndrome: Functional analysis and intervention. Journal of Developmental and Physical Disabilities, 28(2), 267-288. doi: 10.1007/s10882-015-9465-x 


\title{
Repetitive Behavior in Children with Down Syndrome: Functional Analysis and Intervention
}

\author{
Nicole Neil \& Emily A. Jones
}

\section{Suggested citation:}

Neil, N., \& Jones, E. A. (2016). Repetitive behavior in children with Down Syndrome:

Functional analysis and intervention. Journal of Developmental and Physical Disabilities, 28(2), 267-288. doi: 10.1007/s10882-015-9465-x

Acknowledgements: We would like to thank the families who participated in this research. Thank you also to Alysha Rafeeq and Mariya Kishkina for their assistance in data collection. Support for this project was provided by a Doctoral Student Research Grant and a Brain, Cognition, and Behavior Graduate Student Research Grant funded by The City University of New York. 


\begin{abstract}
Children with Down syndrome frequently display repetitive behavior including unusual routines, rituals, and stereotypy. Literature on intervention for repetitive behavior in individuals with Down syndrome frequently includes aversive procedures and interventions not informed by functional assessments. We used an analogue functional analysis to evaluate reinforcers maintaining repetitive behavior in 3 children with Down syndrome. Following identification of automatic functions, we used an $\mathrm{ABAB}$ design and a multiple-baseline design to demonstrate the effectiveness of differential reinforcement of other behavior in reducing repetitive behavior. DRO was effective in decreasing repetitive behavior and, for one participant, repetitive behavior remained low at 1,2, and 3-month follow-up sessions. This study extends current functional analysis methodologies to decrease repetitive behavior for learners with Down syndrome.
\end{abstract} Keywords: Down syndrome, functional analysis, repetitive behavior, differential reinforcement 


\section{Repetitive Behavior in Children with Down Syndrome: Functional Analysis and Intervention}

Repetitive behavior is an umbrella term used to describe behaviors characterized by frequency, repetition, inappropriateness, and invariance (Turner, 1999) including stereotyped and self-injurious behavior. Repetitive behavior is common among individuals with intellectual disability and the prevalence of repetitive behavior is negatively correlated with intellectual ability (McClintock, Hall, \& Oliver, 2003). Some etiologies of intellectual disabilities seem to be associated with repetitive behavior. Children with Down syndrome, for example, frequently display repetitive behavior. Stores, Stores, Fellows, and Buckley (1998) found children with Down syndrome showed significantly greater frequencies of stereotypy as compared to their typically developing siblings and children in the general population. Approximately $40 \%$ of a sample of 3 to 10 year old children with Down syndrome displayed motoric repetitive behavior including hand and finger mannerisms, non-functional play, and unusual sensory interests (Hepburn \& MacLean, 2009). They also found that children with Down syndrome showed unusual preoccupations, routines, and rituals, what might be considered "higher-order" repetitive behaviors. In one study, $70 \%$ of children with Down syndrome showed unusual preoccupations, a significantly higher rate of higher-order repetitive behavior than individuals with a comorbid diagnosis of autism and Down syndrome (Hepburn \& MacLean, 2009). Recent research also suggests that children with Down syndrome are at increased risk for Autism Spectrum Disorders (ASD) relative to children from the general population (Kent, Evans, Paul, \& Sharp, 1999; Lowenthal, Paula, Schwartzman, Brunoni, \& Mercadante, 2007). This diagnosis is associated with greater frequency and severity of restricted and repetitive behaviors (Molloy et al., 2009) 
and high comorbidity with OCD (de Bruin, Ferdinand, Meester, de Nijs, \& Verheij, 2007; Leyfer et al., 2006).

Hollander, Wang, Braun, and Marsh (2009) describe repetitive behaviors on a continuum with motoric repetitive behaviors (e.g. stereotyped movements and self-injury) anchoring the "lower-order" repetitive behaviors to more complex behaviors (e.g., ordering, checking, washing, rituals involving another person) that are routinely observed in persons with Obsessive Compulsive Disorder (OCD) anchoring the "higher-order" repetitive behaviors. While lowerorder behaviors may be "coping" reactions used to modulate stress or arousal, the disruption of higher-order repetitive behaviors often results in anxiety or distress (Hollander et al., 2009). Repetitive behavior classified as "higher-level" are often similar to compulsions seen in OCD with studies (e.g., Mirenda et al., 2010) showing preliminary support for a three-factor model comprised of (a) self-injurious behavior; (b) restricted stereotypic behavior; and (c) compulsive, ritualistic and sameness behaviour using the Repetitive Behavior Scale-Revised (Bodfish, Symons, \& Lewis, 1999).

It appears children with Down syndrome are at risk for both higher and lower order repetitive behavior (Hepburn \& MacLean, 2009; Stores et al., 1998). Repetitive behavior is interfering and associated with additional problem behavior. Evans and Gray (2000) found typically developing children and children with Down syndrome matched on mental age engaged in a similar number of different routinized and compulsive behaviors, but that individuals with Down syndrome engaged in these behaviors with greater frequency and intensity than typically developing children. Additionally, for children with Down syndrome, but not typically developing children, the compulsive behaviors were moderately positively correlated with internalizing and externalizing problem behaviors (Evans \& Gray, 2000). 
In a replication and extension of Evans and Gray (2000), Glenn, Cunningham, and Walsh (2007) also found greater parent-rated intensity of routinized and compulsive behaviors in children with Down syndrome than in typically developing mental age (MA) matched controls, despite similar numbers of routinized and compulsive behaviors across both groups. Further, for young children with DS (with MAs lower than 5 years), routinized and compulsive behaviors were positively correlated with adaptive behaviors; but, for children with MAs over 5 years and young adults with MAs below 5 years, routinized and compulsive behaviors were not correlated with adaptive behaviors, rather with problem behavior. Therefore, as chronological and mental age increased in individuals with Down syndrome, routinized and compulsive behaviors appeared to increase in pathology.

Given the higher rates, intensity, and interference of repetitive behavior and the association with problem behavior among individuals with Down syndrome, there is a need for effective interventions, yet, only a small number of studies have investigated treatment for repetitive behavior in children with Down syndrome, focusing primarily on lower-order repetitive behavior. These interventions targeted loud or repetitive vocalizations (Athens, Vollmer, Sloman, \& Pipkin, 2008; St. Peter et al., 2005;Shaw \& Simms, 2009), tongue protrusion and tongue chewing (Rosine \& Martin, 1983), and motor stereotypy (Anderson, Doughty, Doughty, Williams, \& Saunders, 2010; Rapp, Vollmer, Peter, Dozier, \& Cotnoir, 2004). The most common approaches in these studies were aversive: positive punishment taking the form of a visual cue (Shaw \& Simms, 2009), response blocking (Anderson et al., 2010), and contingent demands (Athens et al., 2008) combined with other procedures.

Investigations of treatment for higher-order repetitive behavior in individuals with Down syndrome are even more limited with most studies using pharmacological interventions. 
Addressing higher-order repetitive behavior in individuals with Down syndrome with pharmacological interventions shows mixed results (Charlot et al., 2002; O'Dwyer et al. 1992; Raitasuo et al., 1996). Some participants are described as improved (Charlot et al., 2002; O’Dwer et al., 1992), while others show partial or no improvement following pharmacological intervention (O’Dwyer et al., 1992; Raitasuo et al., 1996). Thus, alternative approaches to intervening on repetitive behavior in individuals with Down syndrome should be explored.

Functional analysis methodologies have been effectively applied to a variety of problem behavior including repetitive behavior. Using functional analysis to identify the conditions maintaining problem behavior is considered best practice (Hanley, Iwata, \& McCord, 2003) when programming for behavior change. Identifying a behavioral function allows researchers and practitioners to effectively withhold reinforcers maintaining problem behavior, present those reinforcers contingent on appropriate alternative behavior, and alter the reinforcing efficacy of consequent events (Didden, Duker, \& Korzilius, 1997; Hanley et al., 2003). Meta-analyses of the treatment of problem behavior (including repetitive behavior) for individuals with developmental disabilities indicate that treatments preceded by functional analysis in assessing the target behavior are associated with more effective outcomes (Didden et al., 1997; Harvey, Boer, Meyer, \& Evans, 2009).

In one of the few studies involving a functional analysis of repetitive behavior and individuals with Down syndrome, Athens et al. (2008) examined the effects of noncontingent attention, contingent demands, and response cost on reducing the vocal-stereotypy of an 11-yearold boy with a diagnosis of autism and Down syndrome. The results of a functional analysis with four conditions, attention, demand, control, and no-consequence, indicated vocal-stereotypy was 
maintained by automatic reinforcement as indicated by undifferentiated responding and maintenance of the vocal-stereotypy during the no-consequence condition.

Following the functional analysis, Athens et al. (2008) provided a treatment package. Noncontingent attention in the form of social statements provided every $30 \mathrm{~s}$. Academic demands requiring a vocal response were delivered contingent on vocal-stereotypy. Response cost consisted of the child losing access to tangible items if vocal-stereotypy continued to occur following two contingent demands. The treatment package reduced vocal stereotypy to zero and near-zero levels. Athens et al. (2008) also successfully faded the therapist out of the room for 5 minutes during which vocal stereotypy remained at zero occurrences.

The Athens et al. (2008) study illustrates that functional analysis may effectively inform intervention procedures. The author's intervention package still included punishment procedures to reduce the frequency of repetitive behavior. There is evidence that positive procedures alone can reduce the occurrence of repetitive behavior among individuals with Down syndrome. For example, Rosine and Martin (1983) effectively reduced the repetitive tongue protrusion and tongue chewing of two adults with Down syndrome using self-management and differential reinforcement. Positive approaches may also be more acceptable to individuals and families than restrictive or punishment-based procedures (Miltenberger, 1990). Acceptability of intervention is often examined through social validity measures, but none of the studies of repetitive behavior in individuals with Down syndrome collected measures of social validity. While empirical data may indicate improvement, whether the program is helpful can only be determined by the consumer. In order for interventions to be adopted by consumers, they must also perceive them as effective and satisfying. 
Within the literature on the assessment of and intervention for repetitive behavior in Down syndrome there is a need to (1) replicate the use of functional assessment to examine the function of this class of behavior; (2) extend the use of functional assessment to both higher and lower-order repetitive behavior; (3) demonstrate the effectiveness of positive approaches to the treatment of repetitive behavior; and (4) examine the social validity of the intervention package. The current study will, therefore, replicate the use of functional analysis procedures for determining the operant function of repetitive behavior, both lower and higher order behaviors, and implement a differential reinforcement intervention based on the results of this analysis in individuals with Down syndrome.

\section{General Method}

\section{Participants}

Three children with Down syndrome and repetitive behavior participated. Participants were recruited from a local parent group. Participants met the following inclusion criteria: (1) participants' had a diagnosis of Down syndrome, (2) parents identified concerns with repetitive behavior, and (3) participants' parents endorsed one or more forms of repetitive behavior on the Repetitive Behavior Scale -Revised (RBS-R; Bodfish, Symons, \& Lewis, 1999). Two additional participants contacted the first author; one did not meet inclusion criteria and the other dropped out prior to baseline data collection because they had conflicting schedules. Participants' parents completed the Vineland-II (Sparrow, Balla, \& Cicchetti, 2005) at intake (prior to the functional assessment).

Harper was a 6-year-old male with Down syndrome. Harper showed moderate delays with a Full-Scale IQ of $43(<.1$ percentile) on the Stanford-Binet Intelligence Scale, Fifth Edition (Roid, 2003) administered by the first author 1 month prior to the start of the study. He 
scored in the moderately delayed range on the Fluid Reasoning, Knowledge, Quantitative Reasoning, and Visual-Spatial Reasoning $(<.1$ percentile) and in the mildly delayed range on the Working Memory ( $1^{\text {st }}$ percentile) Subscales. On the Vineland-II, Harper scored low in the communication, daily living skills, motor skills, and socialization domains. Harper requested using one-word utterances or gestures and required assistance with daily living tasks including dressing and toileting. Harper came to Queens College weekly for another study in which he was learning expressive and receptive language. Harper's mother identified bruxism, or forceful clenching or grinding of the teeth behavior, to target for assessment and treatment.

Margaret was a 5-year-old female child with Down syndrome. On the Vineland-II, Margaret scored low in the communication, daily living skills, and motor skills domains, and moderately low in the socialization domain. Margaret was capable of following one step instructions and imitating single words. Margaret also came to Queens College weekly for another study involving intervention to teach expressive and receptive language. Margaret received very low scores on the Mullen Scales of Early Learning; her gross motor, visual reception, fine motor, and expressive language score were too low to calculate age equivalent scores. Therefore, age equivalent scores were only calculated for the receptive language subscale, for which Margaret scored at the $1^{\text {st }}$ percentile (T score of 20) with an age equivalent of 19 months. Margaret's mother identified stereotypy, including body rocking, hand and finger, object, and sensory stereotypy as a target for assessment and treatment. These behaviors were targeted together as previous interventions targeting individual topographies were unsuccessful.

Jackie was an 11-year-old female child with Down syndrome and one of 5 children. Jackie attended middle school in a self-contained, $6^{\text {th }}$ grade classroom. On the Vineland-II, Jackie scored low in the communication, daily living skills, and motor skills domains, and 
moderately low in the socialization domain. Jackie followed one and two step instructions, and participated in short conversations. She could read and write short sentences (three to four words) and could perform most daily living tasks without assistance. Jackie's mother identified completeness, specifically door closing as a target during assessment and treatment. Jackie's mother reported that interrupting or blocking door closing resulted in verbal perserveration until the door was closed and that Jackie would interrupt ongoing activities in order to close the door or check that the door was closed.

\section{Setting and Interventionists}

For Harper and Margaret all sessions were conducted in a therapy room at a university. The room contained numerous toys and games, a small table and chairs, bookshelves, and a chalkboard. For Jackie, sessions were conducted in the living room of her home. The living room contained two sofas, a table with various items (e.g., magazines, toys), a piano, a bookcase, and direct view of her bedroom door. The first author served as interventionist for Margaret and Harper; she held a Masters degree, was a Board Certified Behavior Analyst®, and was a Ph.D. student in Psychology. Two research assistants trained in behavioral observation, with an undergraduate degree in psychology assisted with data collection. For Jackie, the first author conducted the functional analysis, and Jackie's mother served as interventionist.

\section{Materials}

The investigator video recorded all functional analysis and intervention sessions to code frequency and latency of target behaviors, intervention integrity, and interobserver agreement. Observers collected data using pens/pencils and datasheets which included the target response definition. 
Adaptive functioning was assessed using the Vineland Adaptive Behavior Scales-II (Sparrow et al., 2005). The Vineland Adaptive Behavior Scales evaluate adaptive functioning in four domains: Communication, Daily Living Skills, Socialization, and Motor Skills. The first author used the "Parent/Caregiver Rating Form"' which is administered as a parent/caregiverreport questionnaire. Parents rate each item as 0 (no, never), 1 (sometimes or partially), 2 (yes, usually), or DK (don't know), although some items may be rated $\mathrm{N}$ (no opportunity).

The Repetitive Behavior Scale-Revised (RBS-R; Bodfish et al., 1999) is a rating scale for measuring the presence and severity of repetitive behaviors. Parents rate each of 43 topographies of repetitive behavior on a 4-point Likert-type scale ranging from (0) behavior does not occur, to (3) behavior occurs and is a severe problem and one item where parents rate the interference of all repetitive behavior, collectively, on a scale from 1 to 100. It has six conceptually derived subscales (Stereotyped Behavior, Self-injurious Behavior, Compulsive Behavior, Ritualistic Behavior, Sameness Behavior, and Restricted Behavior). Internal consistency for the subscales ranges from .78 to .91 (Lam \& Aman, 2007). Inter-rater reliability for the subscales ranges from .57 to .73 when administered for children and adolescents (Lam \& Aman, 2007).

The Consumer Satisfaction Questionnaire is a 'goodness-of-fit' assessment tool (Albin, Lucyshyn, Horner, \& Flannery, 1996) used to evaluate how well the intervention fits with parent's goals, values, and the family's lifestyle. The instrument consists of 20 items that examine five areas relevant to goodness-of-fit: (i) goals and expectations; (ii) support roles; (iii) fit with lifestyle; (iv) implementation effort; and (v) sustainability.

\section{Response Measurement}


Target behaviors were identified by the parent's using the RBS-R, informal observation, and interview with parents. The investigator selected one behavior for assessment and treatment. This behavior was ranked as the highest priority for intervention by the parents. The first author modified response definitions from the RBS-R based on information gathered from interviews and observations to reflect each participant's specific form of the target behavior. Sessions were video recorded so that they could be scored at a later time by two trained undergraduate observers.

Bruxism. For Harper, an observer recorded an occurrence of bruxism when the grinding of upper and lower teeth occurred with sufficient force to create an audible sound. The observer used a partial interval recording procedure during 10-min functional analysis and treatment sessions, divided into 60 intervals. Intervals consisted of $8 \mathrm{~s}$ for observation and $2 \mathrm{~s}$ for recording. She recorded a response if it occurred at any time during the $8 \mathrm{~s}$ interval. If bruxism began in one interval and continued into the next, she counted the response as occurring in both intervals. If bruxism occurred (began and ended) during the $2 \mathrm{~s}$ interval, no response was recorded.

Stereotypy. For Margaret, an observer recorded an occurrence of stereotypy when Margaret displayed repetitive non-functional hand movements (flapping, rubbing, tapping) in the air, against the body, or against objects, rocking (continuous back and forth movement of the torso), mouthing (placing hands or objects in the mouth), or non-functional speech sounds including the grinding of upper and lower teeth. The observer used the same partial interval recording procedures during 10-min functional analysis and treatment sessions as for Harper's bruxism. 
Door closing. For Jackie, an observer recorded the latency (in seconds) to close her bedroom door during 5-min functional analysis and treatment sessions. The observer began recording when the first author opened Jackie's bedroom door and stopped recording when Jackie fully closed her bedroom door. The observer stopped recording after 5 minutes if Jackie did not close her bedroom door.

\section{Overview}

In an initial meeting, the first author obtained consent and assent and parents completed the Vineland-II adaptive behavior assessment to provide descriptive information about adaptive fucntioning, along with two measures of collateral changes associated with the effects of intervention, the Repetitive Behavior Scale-Revised (RBS-R; Bodfish et al., 1999) and The Consumer Satisfaction Questionnaire. Children then participated in the functional analysis and function-based intervention for repetitive behavior. Following intervention, the first author readministered the RBS-R and parents completed the Consumer Satisfaction Questionnaire.

\section{Functional Analyses}

\section{Procedure}

Functional Analysis. The functional analysis conditions were based on the procedures of Iwata, Dorsey, Slifer, Bauman, and Richman (1982/1994). Conditions were alternated in a multielement design. Each condition was presented once per day in random order. Sessions were 10 min long for Margaret and Harper. Jackie's sessions consisted of one trial in which an open door probe was presented, and terminated when Jackie closed the door or when 5 min elapsed.

During the attention condition, the first author and the child sat next to each other. The child had access to toys while the first author pretended to be engaged in work (e.g., reading, 
shuffling papers). The first author responded to repetitive behavior with brief social reprimands (e.g., saying, “Don't do that.”) and statements of concern (e.g., saying, "What's wrong?").

During the demand condition, the first author engaged the child in assembling an inset puzzle. She presented instructional trials for assembling the puzzle on a fixed-time (FT) 30-s schedule. The first author prompted the child using a three-prompt sequence: an initial instruction, followed, if necessary, by a gestural prompt, and then, if necessary, physical guidance. Each occurrence of repetitive behavior resulted in the first author removing the instructional materials, turning away from the child, and allowing $10 \mathrm{~s}$ of escape from the task. If the child displayed repetitive behavior when an instructional trial was about to begin, the trial was postponed until the child stopped.

A no-interaction, rather than a true alone condition, was used for safety reasons. During the no-interaction condition, the child received no activities or consequences for repetitive behavior. The first author was seated in the corner of the room and did not interact with the child. During the control condition, the child had access to preferred activities (e.g., stuffed toys, bubbles, and musical toys). The first author delivered noncontingent attention in the form of brief statements related to the activity in which the child was engaged (e.g., saying, "You're playing the guitar.") every $30 \mathrm{~s}$ and contingent on requests for attention (e.g., if the child brought toys to the first author). The first author provided no differential consequences for repetitive behavior.

For Harper, a tangible condition was added based on first author observations during the course of the previous study in which Harper participated. Prior to each session of the tangible condition, the first author allowed Harper free access to a leisure or food item for a brief time. Items were preferred leisure and food items as identified by Harper's respite worker who 
attended the weekly sessions with Harper (e.g., stuffed toys, bubbles, and musical toys) and based on observations during sessions. When the session began, the first author removed the item, but returned it for $30 \mathrm{~s}$ contingent on the occurrence of repetitive behavior.

Follow-up Functional Analysis (Jackie). As door closing did not occur in the initial functional analysis sessions, we then alternated control, alone, and no-interaction sessions to determine if the first author's presence had a suppressive effect on door closing. During the alone condition, Jackie was alone in the living room while the first author remained in another room for $5 \mathrm{~min}$. Latency was recorded following the session from a video-recording.

\section{Interobserver Agreement}

An undergraduate research assistant independently recorded the frequency of bruxism and stereotypy, and the latency to door closing during $20 \%$ of the functional assessment sessions for each condition to examine interobserver agreement (IOA). For the frequency measures, interval-by-interval IOA was calculated by dividing the number of intervals in which both observers agreed on the occurrence or nonoccurrence of bruxism or stereotypy by the total number of observation intervals and multiplying by 100\%. Mean IOA across functional analysis sessions was $86 \%$ (Range: $80 \%$ - 92\%) for bruxism and $85 \%(80 \%$ - 88\%) for stereotypy. For the latency measure, latency-per-response IOA was calculated by dividing the shorter duration by the longer duration for each occurrence and multiplying by $100 \%$. Mean IOA across functional analysis sessions was $100 \%$ for door closing.

\section{Intervention Integrity}

The same observer who recorded IOA, examined intervention integrity for the functional analysis sessions. Each session was divided into 20, 30 s intervals. Each interval was examined using whole interval recording for the accurate presentation of each component of the assessment 
using a task analysis of the assessment procedures. The independent observer observed the entire $30 \mathrm{~s}$ interval and recorded whether the first author implemented the correct contingency related to the child's bruxism during each $30 \mathrm{~s}$ interval for the functional analysis conditions. For example, during the demand condition, the observer recorded whether the first author presented an instructional trial, prompted child behavior, and implemented a $10 \mathrm{~s}$ escape period contingent on bruxism.

Integrity was calculated for the same $20 \%$ of the sessions for which IOA data were collected. The number of intervals the first author correctly presented each assessment, baseline, and intervention component was divided by the sum of the correct and incorrect intervals, multiplied by 100 , to obtain the percentage of correctly implemented assessment procedures. Integrity was $92 \%$ for Harper, 100\% for Margaret, and 100\% for Jackie.

\section{Results and Discussion}

Figure 1 shows the proportion of intervals with bruxism during the functional analysis with Harper. Following undifferentiated rates of bruxism during the first two functional analysis sessions, bruxism remained lower during the control condition than all other conditions for the remaining 3 sessions. Throughout the functional analysis, bruxism occurred during 0.32 (range $=$ $0.13-0.52$ ) of the control condition intervals, and over 0.50 of the no-interaction, attention, demand, and tangible conditions $(M \mathrm{~s}$ [ranges $]=0.51[0.18-0.75]$ for attention, $0.55[0.4-0.76]$ for alone, $0.54[0.22-0.95]$ for demand, and $0.65[0.26-0.93]$ for tangible). This suggests that Harpers bruxism was maintained by automatic reinforcement

Figure 2 shows the latency to door closing during the functional analysis and follow-up functional analysis with Jackie. Door closing did not occur during the attention, demand, control, and no-interaction conditions during the initial three sessions. We hypothesized the presence of 
another individual may have suppressed the occurrence of door closing. To evaluate this possibility, we conducted a follow-up functional analysis in which we alternated between control, no-interaction, and alone conditions. When the alone condition was introduced, door closing occurred in $0.92 \mathrm{~s}$ and $0.43 \mathrm{~s}$. The short latency to door closing during the alone condition suggested that door closing was maintained by automatic reinforcement and that there was a suppressive effect of the first author's presence.

Figure 3 shows the proportion of intervals with stereotypy during the functional analysis with Margaret. Rates of stereotypy were highest during the no-interaction $(M=0.82[0.67-$ $0.98])$ and demand $(M=0.78[0.65-0.95])$ sessions. This suggests that Margaret's bruxism was maintained by automatic reinforcement, escape from demands, or both.

\section{Treatment Evaluation}

The results from the functional analyses informed individual function-based treatments for each of the three participants. Procedures are presented individually for each participant because each intervention varied as a result of individualizing intervention components.

\section{Design}

A multiple baseline design across participants (Margaret and Jackie) was used to investigate the effectiveness of a multi-component intervention plan including DRO based on the functional analysis. Within this, two treatments were also alternated using a multi-element design for Margaret. The two treatments presented in random order on the same day, once per week. For Harper, a reversal $(\mathrm{ABAB})$ design was used to evaluate the effect of DRO on the frequency of bruxism. A pre-post design was used to evaluate collateral changes associated with the effects of intervention on repetitive behavior (using the RBS-R).

\section{Procedure}


Harper. The results of Harper's functional analysis suggested bruxism was maintained by automatic reinforcement; bruxism occurred at higher rates during the no-interaction, attention, demand, and tangible conditions and during fewer intervals of the control condition. This also suggested that access to attention and tangible items, as occurred during the control condition, could effectively compete with the reinforcing consequences of bruxism.

Baseline. During baseline, the first author engaged Harper in discrete trial teaching opportunities and play. The first author sat across from Harper and provided one-step instructions (e.g., saying, “Open the door.”). She used most-to-least prompting and provided social reinforcers, such as verbal praise and tickles, for correct responses. Interspersed with discrete trial teaching were 5 minutes periods of play with preferred activities (e.g., stuffed toys, bubbles, and musical toys). The first author ignored all occurrences of bruxism during instruction and play.

Intervention. As in baseline, Harper sat at a table with the first author and the first author presented discrete trial teaching opportunities for one-step instructions and 5 minute periods of play. Prompting and reinforcement for one-step instructions during discrete trial instruction remained the same as in baseline.

During intervention, the first author provided reinforcement in the form of attention and $10 \mathrm{~s}$ of access to a toy contingent of the absence of bruxism during the entire DRO interval. Delivery of attention was defined as a brief (1 to $2 \mathrm{~s}$ ) verbal interaction (e.g., saying, "Nice quiet mouth.") between the first author and child; the first author also occasionally patted the Harper's arm or back during the interaction. The first author kept track of the intervals using a digital vibrating countdown timer clipped to her belt, programmed with 2 intervals. The first was the DRO interval and the second was the $10 \mathrm{~s}$ interval for access to the reinforcer. The timer ran 
continuously until the end of the first interval, at which point it vibrated and began a $10 \mathrm{~s}$ interval for access to the reinforcer. Following the $10 \mathrm{~s}$ interval of access to the reinforcer, the timer vibrated and automatically resumed the DRO interval. When bruxism occurred, the first author reset the timer to restart the DRO interval.

For the initial DRO session, we calculated a DRO interval of $50 \%$ of the mean interresponse time (IRT) between instances of bruxism during baseline sessions. The mean IRT during baseline was $34 \mathrm{~s}$; the first DRO interval was set at $17 \mathrm{~s}$. We then progressively increased the mean interval duration by $50 \%$ after every two consecutive sessions in which bruxism remained below $80 \%$ of baseline. We repeated this procedure following the reversal, recalculating a mean IRT during the second baseline (52 s) and an initial DRO interval of $26 \mathrm{~s}$ and continued intervention for an additional 10 sessions.

Follow-up. The first author conducted follow-up sessions 1, 2, and 3 months after the conclusion of DRO treatment. Follow-up procedures were identical to baseline procedures.

Margaret. Margaret's functional analysis suggested that stereotypy was maintained by both automatic reinforcement and escape from demands. However, it is possible that responding in the escape condition was not due to the avoidance or escape contingency but, instead, to the lack of alternative stimulation available during avoidance or escape intervals (Kuhn, DeLeon, Fisher, \& Wilke, 1999). If so, lower levels of responding may have been observed in the control and attention conditions because highly preferred leisure items in the control condition and attention condition competed with stereotypy. During treatment, we conducted an analysis similar to the one conducted by Kuhn et al. (1999) and Rodriguez, Thompson, Schlichenmeyer and Stocco (2012) in which a function-based treatment was matched to one function, but not the 
other to clarify whether stereotypy was maintained by automatic reinforcement and escape from demands or automatic reinforcement alone.

Baseline. Sessions occurred Baseline procedures for Margaret were the same as for Harper except the first author presented instructional trials for expressive instructions rather than one-step instructions on a fixed-time (FT) 30-s schedule.

DRO + Enriched Environment. The DRO + Enriched environment condition was implemented to address the automatic reinforcement function. To enrich the environment, the first author provided Margaret with access to three toys. Toys were selected based on the results of a competing items assessment (Piazza, Adelinis, Hanley, Goh, \& Delia, 2000) in which the first author provided Margaret with 2 minutes to interact with 12 different materials, 6 of which were identified as preferred by her mother and 6 of which provided similar stimulation to Margaret's stereotypy. Margaret was given 2 minutes to interact with each toy and the frequency of stereotypy recorded. The materials were ranked from fewest to largest number of intervals with stereotypy and the top three were selected for treatment. The items used during intervention were: pom poms, a drum, and a singing stuffed toy.

While the toys were available, the first author provided reinforcement in the form of attention and an edible contingent of the absence of stereotypy during the entire DRO interval. DRO procedures were the same for Margaret as for Harper. For the initial DRO session, we calculated a DRO interval of $50 \%$ of the mean interresponse time (IRT) between instances of stereotypy during the final three baseline sessions. The mean IRT during baseline was $21 \mathrm{~s}$; the first DRO interval was set at $11 \mathrm{~s}$. We then progressively increased the mean interval duration by $50 \%$ after every two consecutive sessions in which stereotypy remained below $80 \%$ of baseline. 
DRO + Enriched Environment + Demands. Demands were added to the DRO+Enriched environment condition in an attempt to determine if stereotypy was also maintained by escape from demands. If stereotypy was also maintained by escape from demands, it would persist in the escape condition when attempts to engage in the target behavior resulted in escape, but the other components competed with automatic reinforcement. This condition was identical to the DRO+Enriched Environment condition except the first author presented instructional trials for expressive instructions on a fixed-time (FT) 30-s schedule. At least 12 types of different, yet related, demands were delivered in varied order. All demands required a vocal response. These demands were selected based on data from a previous study indicating Margaret was both accurate and fluent with the required responses. Correct responses were followed by brief praise (e.g., the first author said, 'Good job!'”). Incorrect responses were followed by a model of the correct response (e.g., the first author said, "Say, 'red",'). The first author used a three-prompt sequence: an initial instruction, followed, if necessary, by a partial prompt (an initial consonant sound), and then, if necessary, a full verbal model. Each occurrence of stereotypy resulted in the first author removing the instructional materials, turning away from the child, and allowing $10 \mathrm{~s}$ of escape from the task. If the child displayed stereotypy when an instructional trial was about to begin, the trial was postponed until the child discontinued.

DRO + Enriched Environment + Extinction. During several sessions of the DRO + Enriched environment + demands condition, Margaret showed elevated responding compared to the DRO + Enriched environment condition. This suggested that stereotypy was maintained by both automatic reinforcement and escape from demands. In order to address both the automatic and escape functions, we added escape extinction to the DRO + Enriched environment + 
demands condition. Demands were not removed upon the occurrence of stereotypy in this condition.

Jackie. During Jackie's functional analysis, Jackie displayed door closing only during the alone condition, and not when the first author was present. The short latency to door closing during the alone condition suggested that door closing was maintained by automatic reinforcement and that there was a suppressive effect of the first author's presence.

Baseline. During baseline, Jackie's mother maintained her daily routine. Jackie's mother presented open door probes on days randomly selected by the first author, three times per week. During these probes, Jackie's mother opened Jackie's bedroom door while Jackie was not present. Jackie's mother began video recording when Jackie entered the living room (from which her bedroom door was visible) and stopped video recording when Jackie closed her door, or after 5 min had elapsed.

Interventionist Training. Jackie's mother served as interventionist. The first author trained her by providing her with a typed list of definitions of the components of intervention and verbally describing each of the components. The first author modeled each intervention component, with Jackie's mother playing the role of her child. Jackie's mother closed Jackie's bedroom door while the first author modeled each intervention component three times. The first author then role-played the child closing Jackie's door and Jackie's mother performed each intervention component three times. The first author provided the parent with descriptive spoken feedback immediately following this rehearsal, including positive comments on target components performed correctly and informative corrective feedback on components that were performed incorrectly. Rehearsal and feedback continued with the parent performing three trials and the first author demonstrating three trials until 10 min elapsed. 
Next, the first author modeled the intervention with the child three times. The first author narrated each intervention component while the parent observed. Following this, the parent then performed the intervention with the child three times. The criterion for completion of training was $100 \%$ correct parent responses with the child on two consecutive training trials.

Intervention. The first author developed an individualized multi-component intervention package (Carr \& Carlson, 1993) consisting of strategies focused on: prevention (changing/removing setting events and antecedents) and decreasing target behaviors. At the start of intervention, Jackie's mother told Jackie a rule (or contingency-specifying stimuli which are function-altering; (Baum, 1995; Kunkel, 1997; Schlinger, 1990; Zettle, 1990). She said, "When you are inside your room you can close the door, when you are outside your room the door stays open." Jackie's mother read the rule aloud to Jackie three times per week and presented it visually on Jackie's door.

In addition, Jackie's mother also used DRO, consisting of social positive reinforcement and a tangible contingent on the nonoccurrence of door closing. Jackie's mother offered the choice of tangible items she would earn; she typically chose an edible (e.g., Hot Pockets ®). The initial DRO criteria was set at half of the average baseline (7 s). During the first session Jackie exceeded the criteria by several minutes and reinforcement was faded rapidly. To fade reinforcement the primary author created a visual prompt and token board and Jackie's mother placed it on her door following the first session. The visual showed the days of the week with a box next to them to place a checkmark in. When Jackie followed the rule for the entire day she placed a check-mark in the box. Following three checkmarks Jackie received her pre-selected item. If door closing occurred when Jackie was outside her room, Jackie's mother repeated the rule, and Jackie did not receive a checkmark for that day. 


\section{Interobserver Agreement}

An undergraduate research assistant independently recorded the frequency of bruxism and stereotypy, and the latency to door closing during 29\% (33\% for Harper, 26\% for Margaret, and $28 \%$ for Jackie) of sessions (baseline and intervention) to examine interobserver agreement (IOA). Interobserver agreement procedures were the same as for the functional analyses. Mean IOA across intervention and baseline sessions was 89\% (Range: $80 \%$ - 93\% with one outlier at $70 \%$ ) for bruxism, and $88 \%$ (Range: $87 \%$ - 97\%) for stereotypy. The one session with IOA below $80 \%$ occurred during intervention. During this session, the first author engaged the child in a number of musical activities and the camera was positioned further away from the child. This made coding the proportion of intervals with bruxism from the video recording very difficult, resulting in poor reliability. For the latency measure, latency-per-response IOA was calculated by dividing the shorter duration by the longer duration for each occurrence and multiplying by $100 \%$.

\section{Intervention Integrity}

First author adherence to intervention components was assessed using a procedural checklist of intervention implementation steps. The same observer who recorded IOA, examined intervention integrity. Procedures for assessing integrity were the same as those for functional analysis integrity assessments. Integrity was calculated for the same $33 \%$ for Harper and $26 \%$ for Margaret of the sessions for which IOA data were collected. The number of intervals the first author correctly presented each baseline, and intervention component was divided by the sum of the correct and incorrect intervals, multiplied by 100 , to obtain the percentage of correctly implemented assessment procedures. Integrity was $95 \%$ for Harper, and 94\% for Margaret. 
For Jackie, the same observer who recorded IOA, examined parental adherence to intervention components. Parental adherence to baseline and intervention components was assessed using a procedural checklist of intervention implementation steps. Intervention integrity checks were completed from video recordings for a random selection of $28 \%$ of sessions (baseline and intervention) by the same research assistant performing IOA. For intervention sessions, the observer recorded if Jackie's mother stated the rule, ignored the occurrence of door closing, and delivered attention and a checkmark following the appropriate interval with no door closing. For baseline sessions, the observer recorded if Jackie's mother presented the open door probe and ignored door closing behavior. Parental adherence was $100 \%$.

The first author's adherence to interventionist training was also assessed using a procedural checklist of training implementations steps. The same observer who recorded IOA completed these from a video recording of the training sessions and integrity was $100 \%$.

\section{Results and Discussion}

Harper's intervention. Figure 4 shows the results of Harper's treatment evaluation. During the initial baseline, bruxism occurred during 0.39 (range: $0.32-0.43$ ) of the intervals. The dashed mastery line represents an $80 \%$ decrease from baseline in the proportion of intervals with bruxism. An immediate decrease in the number of intervals with bruxism occurred when the DRO was introduced. We gradually faded the DRO interval to $39 \mathrm{~s}$ with near zero levels $(M=$ 0.02 , range $=0.00-0.10)$ of bruxism prior to implementing the reversal. Return to baseline conditions resulted in an increase in bruxism $(M=0.22)$. Re-introduction of the DRO condition again resulted in reduction in the proportion of intervals with bruxism $(M=.037$, range $=0.00-$ 
0.12). The DRO intervals were faded to $87 \mathrm{~s}$ and reductions in the number of intervals with bruxism maintained at 1-, 2- and 3-month follow-up.

Margaret and Jackie's Intervention. Figure 5 shows the results of Jackie's and Margaret's intervention. For Jackie, prior to intervention, the mean latency to door closing was $14.66 \mathrm{~s}$ (range: $12-17 \mathrm{~s}$ ). Following the introduction of the multi-component intervention, Jackie's door closing did not occur and sessions terminated following 5 min. Jackie's mother also anecdotally reported that door closing did not occur outside of the probes.

During baseline, Margaret displayed stereotypy during .68 of the intervals (range $=.26$ to 95). Following introduction of intervention to eliminate the automatic reinforcer, levels of stereotypy decreased across both DRO and enriched environment and DRO and enriched environment plus escape conditions (DRO + Enriched: $M=.25, \mathrm{DRO}+$ Enriched + Demands: $M$ $=.19)$. Responding was elevated in several of the demand sessions, suggesting that escape from demands may have also functioned as a reinforcer for stereotypy. When escape extinction was introduced, stereotypy remained at similar levels $(M=.33)$ disconfirming this hypothesis and suggesting that stereotypy was in fact maintained by automatic reinforcement.

Social Validity. Pre- and post-intervention scores on the RBS-R are presented in Table 1. Scores on the expected subscales for Harper and Jackie (stereotyped and compulsive, respectively) decreased. For Margaret, her mother indicated increases in stereotypy from pre- to post-intervention. For Margaret and Jackie, parent's indicated lower levels of interference from their repetitive behavior at post-intervention. For Harper, interference was greater at postintervention than at pre-intervention. Finally, the Goodness of fit questionnaire revealed a high level of satisfaction with the treatment with a mean score of 81.33 out of 100 . Overall, families 
perceived the intervention plans and their use to be sustainable, or a good fit with the family routines and family life in general.

\section{General Discussion}

The current study replicated the use of functional analysis procedures for determining the operant function of repetitive behavior, both higher- and lower-order repetitive behaviors, and demonstrated the effectiveness of a differential reinforcement intervention based on the results of this analysis, all with high intervention integrity and social validity. The current study extended previous research by investigating an assessment and intervention for repetitive behavior in children with Down syndrome.

Results demonstrate the application of functional assessment of repetitive behavior in children with Down syndrome for both higher order and lower order repetitive behavior. While functional approaches to problem behavior emphasize function over form in designing interventions, response topography should not be neglected. Increasingly, researchers differentiate repetitive behavior as "higher level" (e.g., washing, rituals involving others, rigid routines, ordering and arranging) and "lower level" (e.g., restricted stereotypy, self-injury; Hollander et al., 2009; Turner, 1999). Factor analyses of the RBS-R subscales suggest a distinction between these lower and higher-order repetitive behaviors (e.g., Mirenda et al., 2010). Support for a distinction between these two classes can also be seen in the results of this study. Harper and Margaret's behaviors fell within the stereotypy subscale (lower-order) and Jackie's within the compulsive or ritualistic subscales (higher-order) on the RBS-R. Higher order repetitive behavior, especially insistence on sameness behavior (e.g., keeping a particular door closed) strongly resembles typical OCD-related "just right" behaviors, which usually occur in response to an obsession or the imposition of rigidly applied 'rules' (DSM-5, American 
Psychiatric Association [APA], 2013). These observations are supported by factor analyses of the RBS-R, with compulsive, ritualistic and sameness behaviour making up one factor in a threefactor model (Mirenda et al., 2010). During intervention Jackie showed rapid decreases in the behavior upon introduction of the rule prior to contacting the DRO contingency. The most likely explanation for this finding is that the verbal description of the intervention plan given to Jackie was sufficient to influence her behavior as a form of rule-governed mediation.

Despite varying topographies of repetitive behavior all three individuals demonstrated similar functions. Previous studies with children with Down syndrome and repetitive behavior either did not attempt to identify reinforcers maintaining repetitive behavior or relied on descriptive assessments of function. The results of the functional analysis are consistent with other functional assessments of repetitive behavior. A substantial body of research provides evidence for a sensory function of stereotypy, whereby behavior is maintained by automatic reinforcement (Lovaas, 1987; Piazza et al., 2000; Rapp, 2006; Rincover, 1978; Rincover, Cook, Peoples, \& Packard, 1979). There is also evidence that "higher-level" repetitive behavior or ritualistic behavior can be maintained by automatic reinforcement among children with developmental disabilities (Rodriguez et al., 2012).

Despite identifying an automatic function, this study did not include a follow-up assessment to determine whether negative or positive reinforcement contingencies. Repetitive behavior could serve to produce a pleasurable sensory stimulus or serve to remove unpleasant sensory stimuli (e.g., removal of anxiety). Since this follow-up was not included the effectiveness of the intervention, DRO, depended on the ability of the alternative reinforcer to compete with the unknown variable maintaining the participants' behavior. Future research 
should consider presenting follow-up analyses to identify which of these contingencies maintains repetitive behavior in individuals with Down syndrome.

The function identified in this study is consistent with other assessments of repetitive behavior; it is less characteristic of the functions of problem behavior that tend to be described among individuals with Down syndrome. Individuals with Down syndrome have been described as showing a pattern of problem behavior to obtain attention or escape from task demands (Feeley \& Jones, 2006). Typical problem behavior is described as either charming off-task behaviors serving to engage other individuals and obtain social attention, or avoidance behaviors that interfere with task completion (Fidler, 2005; Wishart, 1993). These results reinforce the recommendation for functional analysis and function-based interventions as function may not be clear from diagnosis or response topography alone.

As illustrated in this study, individuals with Down syndrome also display problem behavior that falls outside of what is typically described in the Down syndrome behavior phenotype and this behavior may serve functions that are not typically described in individuals with Down syndrome. There is little information regarding repetitive behavior among individuals with Down syndrome, likely because it is not a diagnostic feature. As researchers begin to develop interventions targeting behaviors within the Down syndrome phenotype, they must also consider the unique contributions of Down syndrome to less frequently displayed behaviors.

Results from the differential reinforcement intervention demonstrate the effectiveness of reinforcement-based approaches and that multiple techniques can be used to address repetitive behavior serving similar functions. We also demonstrated that the gains from this intervention can be maintained up to 3-months following intervention for Harper. Reinforcement-based procedures are preferred over aversive or punishment procedures as preventative rather than 
reactive strategies. In contrast to prescriptive approaches, function-based interventions allow greater opportunity for interventionists to tailor intervention to meet the specific needs or preferences of the client. Additionally, greater visibility and availability of effective reinforcement-based procedures may decrease the reliance on aversive procedures. Future research might compare alternative approaches to addressing similar functions of repetitive behavior to maximize decreases in rates of repetitive behavior.

We demonstrated decreases in repetitive behavior for Jackie and Margaret using a multiple-baseline across participants design. Repetitive behavior for both children showed rapid decreases upon introduction of the intervention, though the strength of this design in demonstrating the effect of DRO is limited by differences in the topography and individualized components of intervention across lags of the multiple-baseline design. We suggest the immediate changes in level of repetitive behavior following the introduction of intervention provide evidence a functional relationship, nevertheless future research should replicate these results using designs with greater control.

This study had a number of strengths, including measures of social validity and intervention integrity. Social validity and intervention integrity were high for both the functional analysis and intervention. Consumer satisfaction, or goodness-of-fit, was highest for Jackie, whose intervention was conducted by her mother and within the home. Adapting the intervention for delivery by Jackie's mother did not appear to compromise the efficacy of the intervention, and may have helped to incorporate family values, beliefs, and goals into the intervention, enhancing consumer satisfaction. This is consistent with the intervention literature on repetitive behavior in individuals with other developmental disabilities; involving key individuals, such as parents and teachers, not only results in high social validity, but also helps maintain the positive 
results of treatment (Patterson, Smith, \& Jelen, 2010). Despite this, only a minority of studies includes intervention for problem behavior delivered by parents (Harvey et al., 2009).

The results on the RBS-R showed decreases in the interference of repetitive behavior for two of the participants (Margaret and Jackie), and decreases on the relevant subscale for two of the participants (Harper and Jackie). The RBS-R is typically used in pharmacological research on repetitive behaviors in individuals with intellectual disability and the sensitivity of the RBS-R may not be sufficient for such narrow intervention plans as presented in this study. We included the RBS-R as it is a well-established measure of repetitive behavior among individuals with developmental disability, but future research may want to consider developing or using measures of symptom severity that are tailored to individualized behavior plans. It is impressive then, considering these potential sensitivity issues, that we found such large decreases in interference for two of the participants.

The findings of this study add to the growing literature demonstrating the use of behavior analytic strategies to address problem behavior in children with Down syndrome (Feeley \& Jones, 2006). While repetitive behavior is not considered a defining characteristic of Down syndrome, many individuals with Down syndrome display some form of repetitive behavior (e.g., bruxism, rocking, mouthing; Evans \& Gray, 2000). Decreasing repetitive behavior among individuals with Down syndrome may afford them with greater numbers of learning opportunities and decrease social stigmatization and, thus, should be considered an important target for increasing the quality of life of individuals with Down syndrome. 


\section{References}

Albin, R. W., Lucyshyn, J. M., Horner, R. H., \& Flannery, K. B. (1996). Contextual fit for behavioral support plans: A model for “goodness of fit.”.Positive behavioral support: Including people with difficult behavior in the community, 8, 98.

American Psychiatric Association (2013). Diagnostic and Statistical Manual of Mental Disorders: DSM 5. Arlington, VA: American Psychiatric Association.

Anderson, C. M., Doughty, S. S., Doughty, A. H., Williams, D. C., \& Saunders, K. J. (2010). Evaluation of stimulus control over a communication response as an intervention for stereotypical responding. Journal of Applied Behavior Analysis, 43(2), 333-339.

Athens, E. S., Vollmer, T. R., Sloman, K. N., \& Pipkin, C. S. P. (2008). An analysis of vocal stereotypy and therapist fading. Journal of Applied Behavior Analysis, 41(2), 291-297.

Baum, W. M. (1995). Rules, culture, and fitness. The Behavior Analyst, 18(1), 1-21.

Bodfish, J. W., Symons, F. W., \& Lewis, M. H. (1999). The Repetitive Behavior ScaleRevised, Western Carolina Center Research Reports.

Carr, E. G., \& Carlson, J. I. (1993). Reduction of severe behavior problems in the community using a multicomponent treatment approach. Journal of Applied Behavior Analysis, 26, $157-$ 172.

Charlot, L., Fox, S., \& Friedlander, R. (2002). Obsessional slowness in Down's syndrome. Journal of Intellectual Disability Research, 46(6), 517-524.

de Bruin, E. I., Ferdinand, R. F., Meester, S., de Nijs, P. F., \& Verheij, F. (2007). High rates of psychiatric co-morbidity in PDD-NOS. Journal of Autism and Developmental Disorders, $37(5), 877-886$. 
Didden, R., Duker, P. C., \& Korzilius, H. (1997). Meta-analytic study on treatment effectiveness for problem behaviors with individuals who have mental retardation. American Journal on Mental Retardation, 101(4), 387-399.

Evans, D. W., \& Gray, F. L. (2000). Compulsive-like behavior in individuals with Down syndrome: Its relation to mental age level, adaptive and maladaptive behavior. Child Development, 71(2), 288-300.

Feeley, K., \& Jones, E. (2006). Addressing challenging behaviour in children with Down syndrome: The use of applied behaviour analysis for assessment and intervention. Down Syndrome Research and Practice, 11(2), 64-77.

Fidler, D. J., Philofsky, A., Hepburn, S. L., \& Rogers, S. J. (2005). Nonverbal requesting and problem-solving by toddlers with Down syndrome. Journal Information, 110(4).

Glenn, S., Cunningham, C., \& Walsh, K. (2007). Typical or pathological? Routinized and compulsive-like behaviors in children and young people with down syndrome. Intellectual and Developmental Disabilities, 45(4), 246-256.

Hanley, G. P., Iwata, B. A., \& McCord, B. E. (2003). Functional analysis of problem behavior: A review. Journal of Applied Behavior Analysis, 36, 147-185.

Harvey, S. T., Boer, D., Meyer, L. H., \& Evans, I. M. (2009). Updating a meta-analysis of intervention research with challenging behaviour: Treatment validity and standards of practice. Journal of Intellectual and Developmental Disability, 34(1), 67-80.

Hepburn, S. L., \& MacLean, W. E. (2009). Maladaptive and repetitive behaviors in children with down syndrome and autism spectrum disorders: Implications for screening. Journal of Mental Health Research in Intellectual Disabilities, 2(2), 67-88. 
Hollander, E., Wang, A. T., Braun, A., \& Marsh, L. (2009). Neurological considerations: Autism and Parkinson's disease. Psychiatry Research, 170(1), 43-51.

Iwata, B. A., Dorsey, M. F., Slifer, K. J., Bauman, K. E., \& Richman, G. S. (1982). Toward a functional analysis of self-injury. Analysis and Intervention in Developmental Disabilities, 2(1), 3-20.

Iwata, B. A., Dorsey, M. F., Slifer, K. J., Bauman, K. E., \& Richman, G. S. (1994). Toward a functional analysis of self-injury. Journal of Applied Behavior Analysis, 27, 197-209.

Kent, L., Evans, J., Paul, M., \& Sharp, M. (1999). Comorbidity of autistic spectrum disorders in children with Down syndrome. Developmental Medicine \& Child Neurology, 41(3), 153158.

Kuhn, D. E., DeLeon, I. G., Fisher, W. W., \& Wilke, A. E. (1999). Clarifying an ambiguous functional analysis with matched and mismatched extinction procedures. Journal of Applied Behavior Analysis, 32, 99-102.

Kunkel, J. H. (1997). The analysis of rule-governed behavior in social psychology. Psychological Record, 47(4), 699-716.

Lam, K. S., \& Aman, M. G. (2007). The repetitive behavior scale-revised: Independent validation in individuals with autism spectrum disorders. Journal of Autism and Developmental Disorders, 37(5), 855-866.

Leyfer, O. T., Folstein, S. E., Bacalman, S., Davis, N. O., Dinh, E., Morgan, J., . . Lainhart, J. E. (2006). Comorbid psychiatric disorders in children with autism: Interview development and rates of disorders. Journal of Autism and Developmental Disorders, 36(7), 849-861.

Lovaas, O. I. (1987). Behavioral treatment and normal educational and intellectual functioning in young autistic children. Journal of Consulting and Clinical Psychology, 55(1), 3. 
Lowenthal, R., Paula, C. S., Schwartzman, J. S., Brunoni, D., \& Mercadante, M. T. (2007).

Prevalence of pervasive developmental disorder in down's syndrome. Journal of Autism and Developmental Disorders, 37(7), 1394-1395.

McClintock, K., Hall, S., \& Oliver, C. (2003). Risk markers associated with challenging behaviours in people with intellectual disabilities: A meta-analytic study. Journal of Intellectual Disability Research, 47(6), 405-416.

Miltenberger, R. G. (1990). Assessment of Treatment Acceptability A Review of the Literature. Topics in Early Childhood Special Education, 10(3), 24-38.

Mirenda, P., Smith, I. M., Vaillancourt, T., Georgiades, S., Duku, E., Szatmari, P., .. . Volden, J. (2010). Validating the repetitive behavior scale-revised in young children with autism spectrum disorder. Journal of Autism and Developmental Disorders, 40(12), 1521-1530.

Molloy, C., Murray, D., Kinsman, A., Castillo, H., Mitchell, T., Hickey, F., \& Patterson, B. (2009). Differences in the clinical presentation of trisomy 21 with and without autism. Journal of Intellectual Disability Research, 53(2), 143-151.

O'Dwyer, J., Holmes, J., \& Collacott, R. A. (1992). Two cases of obsessive-compulsive disorder in individuals with Down's syndrome. Journal of Nervous and Mental Disease.

Patterson, S. Y., Smith, V., \& Jelen, M. (2010). Behavioural intervention practices for stereotypic and repetitive behaviour in individuals with autism spectrum disorder: a systematic review. Developmental Medicine \& Child Neurology, 52(4), 318-327.

Piazza, C. C., Adelinis, J. D., Hanley, G. P., Goh, H., \& Delia, M. D. (2000). An evaluation of the effects of matched stimuli on behaviors maintained by automatic reinforcement. Journal of Applied Behavior Analysis, 33(1), 13-27. 
Raitasuo, S., Virtanen, H., \& Raitasuo, J. (1998). Anorexia nervosa, major depression, and obsessive-compulsive disorder in a Down's syndrome patient.International Journal of Eating Disorders, 23(1), 107-109.

Rapp, J. T. (2006). Toward an empirical method for identifying matched stimulation for automatically reinforced behavior: A preliminary investigation. Journal of Applied Behavior Analysis, 39, 137-140.

Rapp, J. T., Vollmer, T. R., Peter, C., Dozier, C. L., \& Cotnoir, N. M. (2004). Analysis of response allocation in individuals with multiple forms of stereotyped behavior. Journal of Applied Behavior Analysis, 37, 481-501.

Rincover, A. (1978). Sensory extinction: A procedure for eliminating self-stimulatory behavior in developmentally disabled children. Journal of Abnormal Child Psychology, 6(3), 299310.

Rincover, A., Cook, R., Peoples, A., \& Packard, D. (1979). Sensory extinction and sensory reinforcement principles for programming multiple adaptive behavior change. Journal of Applied Behavior Analysis, 12(2), 221-233.

Rodriguez, N. M., Thompson, R. H., Schlichenmeyer, K., \& Stocco, C. S. (2012). Functional analysis and treatment of arranging and ordering by individuals with an autism spectrum disorder. Journal of Applied Behavior Analysis, 45, 1-22.

Roid, G. (2003). Stanford-Binet Intelligence Scales (SB5). Rolling Meadows, IL: Riverside,

Rosine, L. P., \& Martin, G. L. (1983). Self-management training to decrease undesirable behavior of mentally handicapped adults. Rehabilitation Psychology, 28(4), 195.

Schlinger, H. D., Jr. (1990). A reply to behavior analysts writing about rules and rule-governed behavior. The Analysis of Verbal Behavior, 8, 77-82. 
Shaw, R., \& Simms, T. (2009). Reducing attention-maintained behavior through the use of positive punishment, differential reinforcement of low rates, and response marking. Behavioral Interventions, 24(4), 249-263.

Sparrow, S. S., Balla, D. A., \& Cicchetti, D. V. (2005). Vineland-II Adaptive Behavior Scales, Second Edition. San Antonio, TX: Pearson Assessments.

St. Peter, C. C., Vollmer, T. R., Bourret, J. C., Borrero, C. S., Sloman, K. N., \& Rapp, J. T. (2005). On the role of attention in naturally occurring matching relations. Journal of Applied Behavior Analysis, 38(4), 429-443.

Stores, R., Stores, G., Fellows, B., \& Buckley, S. (1998). Daytime behaviour problems and maternal stress in children with Down's syndrome, their siblings, and non-intellectually disabled and other intellectually disabled peers. Journal of Intellectual Disability Research, 42(3), 228-237.

Turner, M. (1999). Annotation: Repetitive behaviour in autism: A review of psychological research. Journal of Child Psychology and Psychiatry, 40(6), 839-849.

Wishart, J. (1993). Learning the hard way: Avoidance strategies in young children with Down syndrome. Down Syndrome Research and Practice, 1(2), 47-55.

Wolf, M. M. (1978). Social validity: The case for subjective measurement, or how applied behavior analysis is finding its heart. Journal of Applied Behavior Analysis, 1, 203-214.

Zettle, R. D. (1990). Rule-governed behavior: A radical behavioral answer to the cognitive challenge. The Psychological Record, 40, 41-49. 
Table 1.

Pre-and Post-Treatment Scores on the Repetitive Behavior Scale-Revised.

\begin{tabular}{|c|c|c|c|c|c|c|c|c|c|c|c|c|c|c|c|}
\hline \multirow[b]{3}{*}{ Participant } & \multicolumn{14}{|c|}{ RBS-R } & \multirow{3}{*}{$\begin{array}{c}\text { Goodness- } \\
\text { of-Fit }\end{array}$} \\
\hline & \multicolumn{2}{|c|}{ Stereotyped } & \multicolumn{2}{|c|}{ Self-Injurious } & \multicolumn{2}{|c|}{ Compulsive } & \multicolumn{2}{|c|}{ Ritualistic } & \multicolumn{2}{|c|}{ Sameness } & \multicolumn{2}{|c|}{ Restricted } & \multicolumn{2}{|c|}{ Interference } & \\
\hline & Pre & Post & Pre & Post & Pre & Post & Pre & Post & Pre & Post & Pre & Post & Pre & Post & \\
\hline Harper & 5 & 3 & 0 & 0 & 2 & 3 & 2 & 5 & 7 & 7 & 0 & 2 & 20 & 35 & 80 \\
\hline Margaret & 7 & 8 & 0 & 3 & 4 & 4 & 2 & 3 & 1 & 3 & 0 & 0 & 40 & 10 & 71 \\
\hline Jackie & 1 & 1 & 0 & 0 & 5 & 1 & 3 & 0 & 0 & 0 & 1 & 0 & 25 & 1 & 97 \\
\hline
\end{tabular}




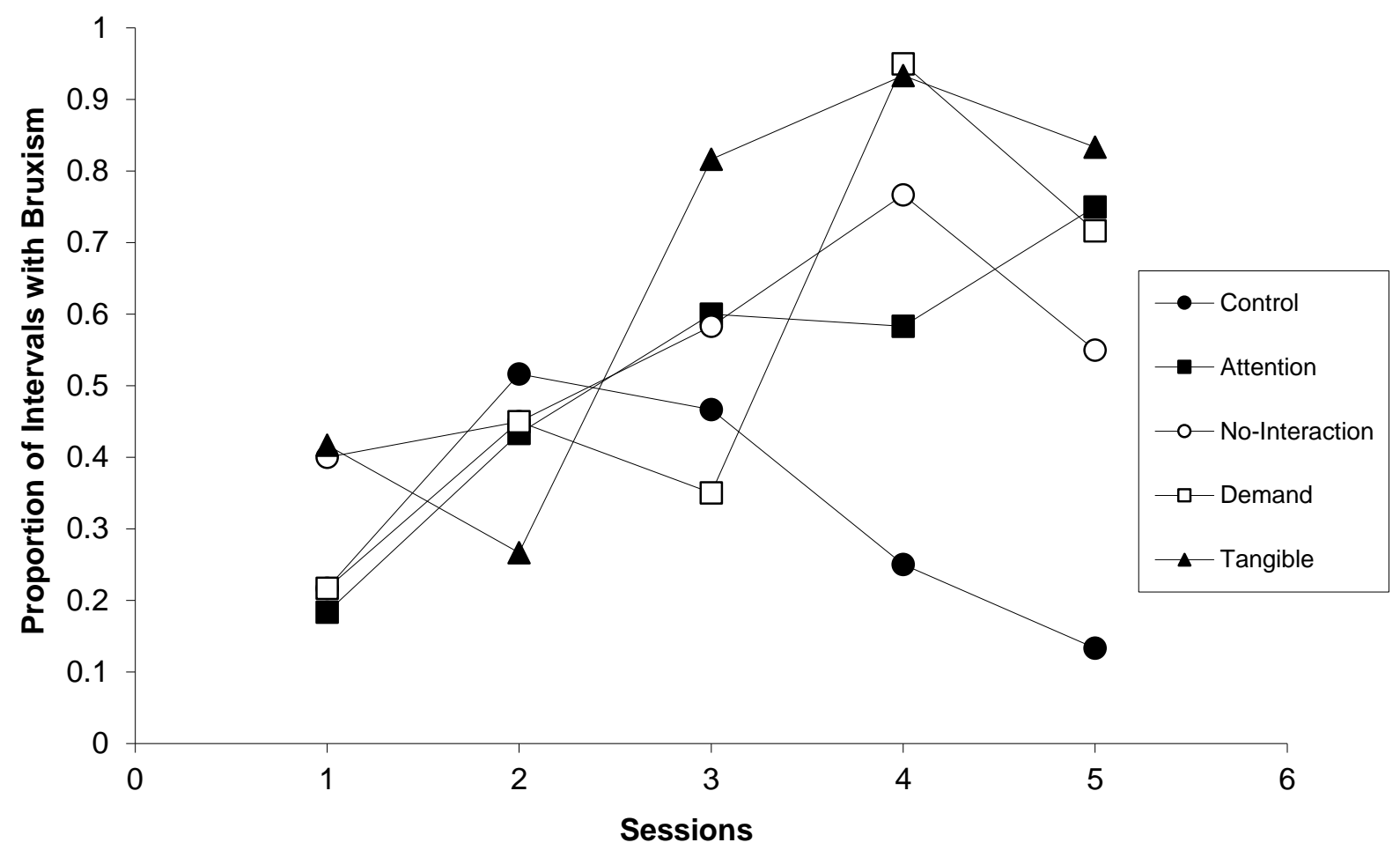

Figure 1. Harper's functional analysis showing the proportion of intervals with bruxism per session during each of the five functional analysis conditions. 


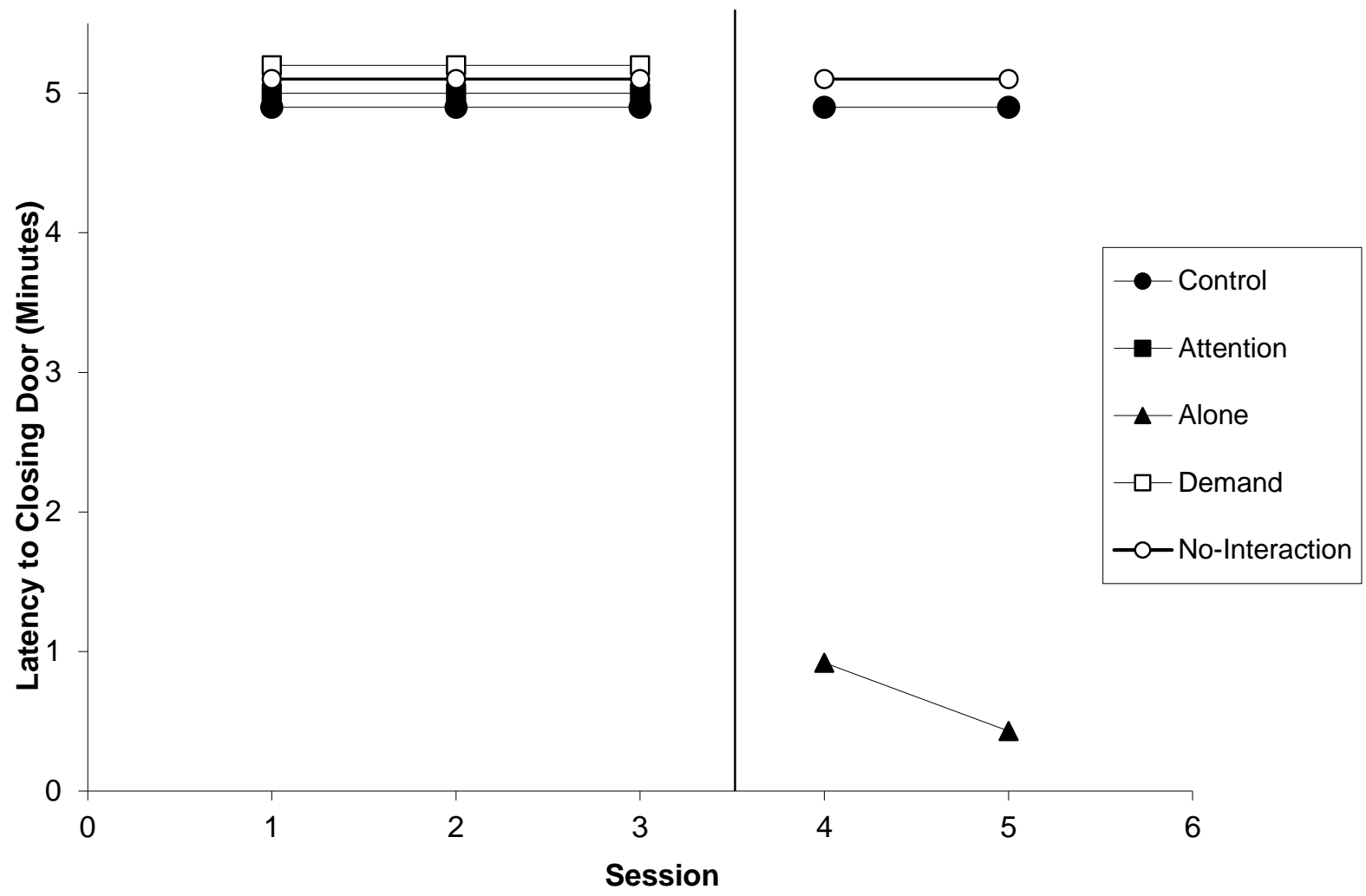

Figure 2. Jackie's functional analysis showing the latency to door closing in each session during each of the five functional analysis conditions. 


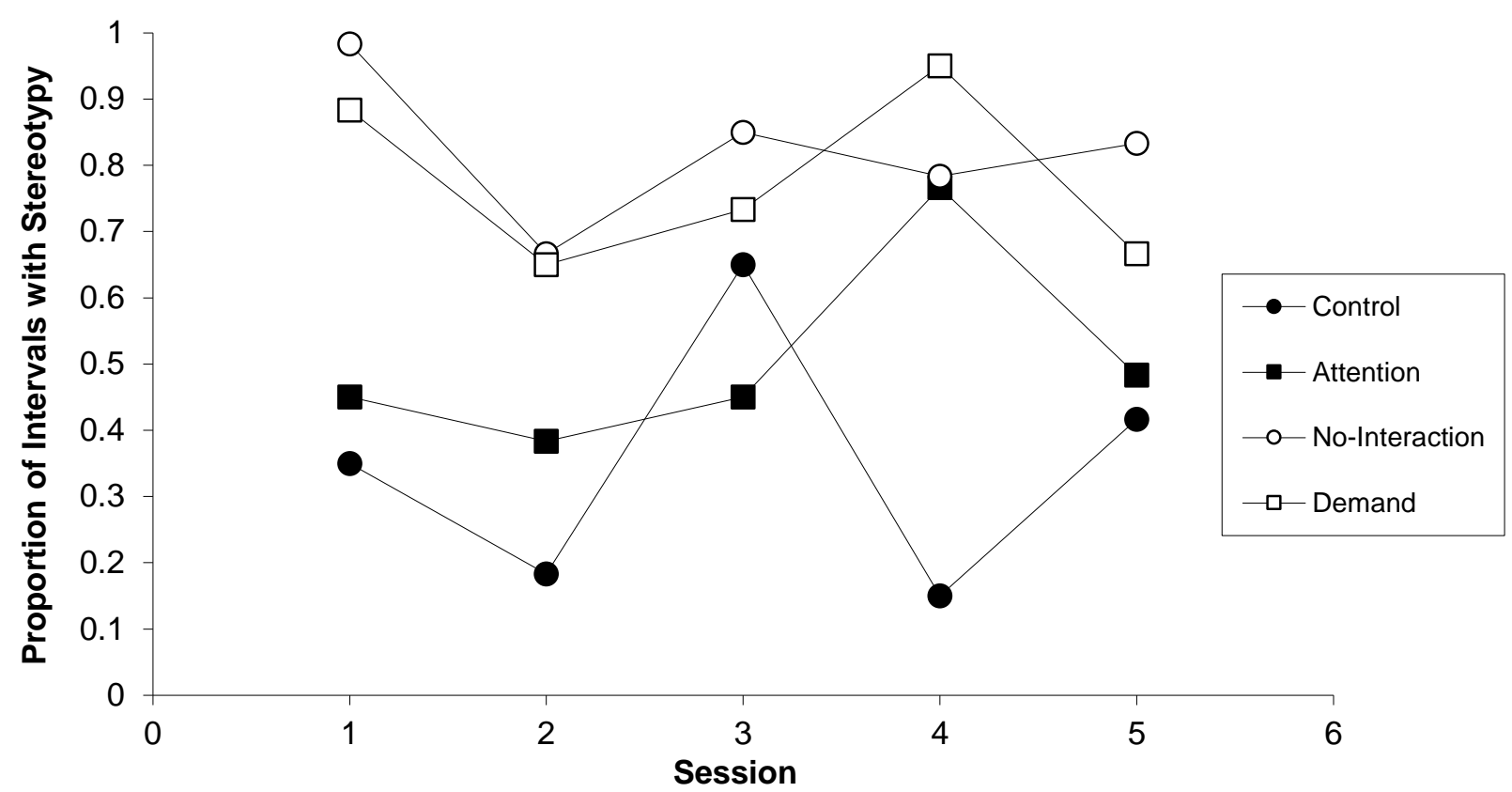

Figure 3. Margaret's functional analysis showing the proportion of intervals with sterotypy per session during each of the four functional analysis conditions. 


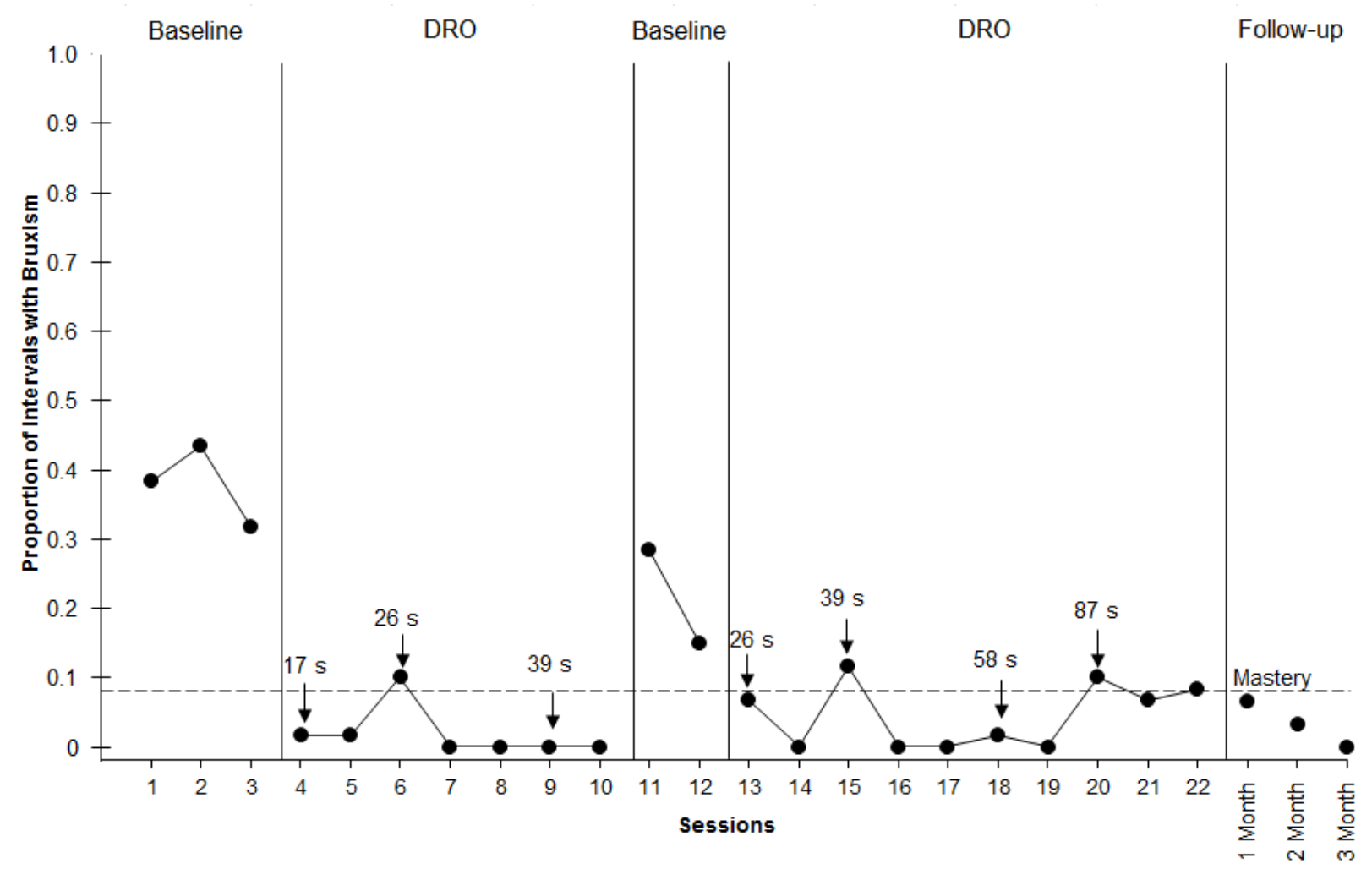

Figure 4. Proportion of intervals with bruxism during basline, DRO intervention, and 1-, 2-, 3month follow-up. The numbers above each data point indicate the DRO interval duration (in seconds). The mastery line represents an $80 \%$ decrease from baseline in the proportion of intervals with bruxism. 


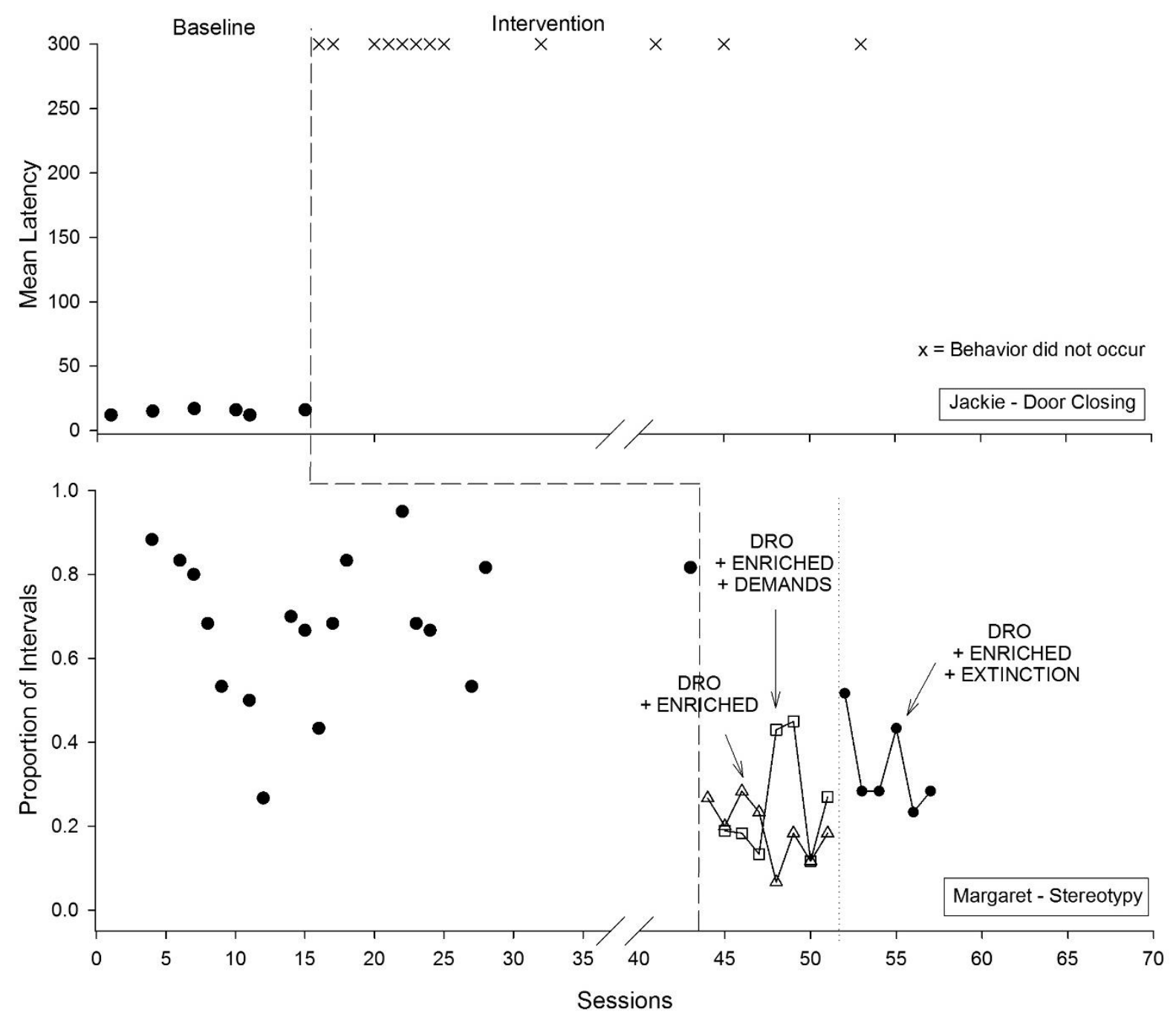

Figure 5. Latency to door closing for Jackie (top panel) and proportion of intervals with stereotypy for Margaret (bottom panel) during baseline, and intervention. 